Zapatero Ayuso, J.A., González Rivera, M.D. \& Campos Izquierdo, A. (2017). Diseño y valoración de una investigación evaluativa. La enseñanza por competencias en Educación Física. Revista Electrónica Interuniversitaria de Formación del Profesorado, 20(1), 19 -34.

\title{
Diseño y valoración de una investigación evaluativa. La enseñanza por competencias en Educación Física
}

\author{
Jorge Agustín Zapatero Ayuso 1, María Dolores González Rivera 2, Antonio Campos \\ Izquierdo 3 \\ 1 Universidad Complutense de Madrid, 2 Universidad de Alcalá, 3 Universidad Politécnica de \\ Madrid
}

\section{Resumen}

El propósito de este artículo es dar a conocer el desarrollo de una investigación evaluativa, empleada para valorar la aplicación del modelo competencial, y la valoración que hacen de este proceso sus participantes: un grupo de profesores de Educación Física de la Comunidad Autónoma de Madrid en Secundaria. Esta investigación fue desarrollada desde la complementariedad metodológica, cualitativa y cuantitativa, aplicando diversas técnicas e instrumentos en cuatro fases sucesivas: preliminar, de desarrollo, de continuidad y de valoración. Se exponen los resultados de la metaevaluación de los participantes, desarrollada en la fase de valoración. Los resultados muestran como este método de investigación fue valorado positivamente y conllevó cambios en el desempeño y la formación de los docentes para enseñar por competencias. La presentación de este estudio pretende favorecer la replicabilidad del mismo para la exploración del objeto de investigación en contextos similares o la exploración de nuevos problemas.

\section{Palabras clave}

Competencias clave; investigación evaluativa; formación del profesorado; educación secundaria.

\section{Contacto:}

Jorge Agustín Zapatero Ayuso, jorge.zapatero.a@gmail.com, Rector Royo Villanova s/n, Ciudad Universitaria, 28040 - MADRID. 


\title{
Design and assessment of evaluative research. Teaching competences in physical education
}

\begin{abstract}
The aim of this paper is to present the development of an evaluative research, used to assess the implementation of competency model, and the valuation that make these process participants: a group of teachers of Physical Education of the Comunidad Autónoma de Madrid in Secondary Education. This research was developed from the methodological complementarity, qualitative and quantitative, applying various techniques and instruments in four stages: preliminary, development, continuity and valuation. Results of the meta-evaluation of participants, developed in the assessment phase, are discussed. The results show how this research method was assessed positively and led to changes in performance and training of teachers to teach competency. The presentation of this project aims to promote the applicability thereof to scan the object of study in similar contexts or exploring new research problems.
\end{abstract}

\section{Key words}

Key competencies; evaluative research; teacher training; secondary education.

\section{Introducción}

La investigación evaluativa ha surgido como un método que comprende la intervención educativa como una acción crítica para mejorar la misma (Tejedor, 2000). Para Lukas y Santiago (2004), la investigación evaluativa es:

"el proceso de identificación, recogida y análisis de información relevante, que podría ser cuantitativa o cualitativa, de manera sistemática, rigurosa, planificada, dirigida, objetiva, creíble, fiable y válida para emitir juicios de valor basados en criterios y referencias preestablecidos para determinar el valor y el mérito del objeto educativo en cuestión a fin de tomar decisiones que ayuden a optimizar el mencionado objeto" (p.84).

El método evaluativo supone una herramienta para la mejora continua de la calidad de programas y de los individuos incluidos en él (Martínez-Mediano, 2004). Por ende, esta mejora continua de las programaciones evaluadas beneficia al personal implicado, el profesorado, y, de modo indirecto, al cuerpo social en que se encuentra inmerso, sus alumnos (Pérez-Juste, 2000).

En cuanto a la evaluación del profesorado, es uno de los ámbitos más importantes en la actualidad y juega un papel innegable en la mejora de la escuela (Lukas y Santiago, 2004). En cuanto al alumnado, la investigación evaluativa ayuda a que participen en programas más adecuados $y$, en lo que se refiere al problema de este estudio, más cercanos a las necesidades y exigencias de la nueva sociedad del siglo XXI: el desarrollo de competencias y el aprendizaje a lo largo de la vida (Castejón, 1996; Ramírez, 2015).

Al respecto, este modelo educativo posee un amplio alcance y se ha adoptado en numerosos sistemas educativos y niveles de enseñanza. Se ha erigido como el paradigma educativo imperante en Europa, sirva como referencia el informe de European Commission, EACEA y Eurydice (2012), que explica y analiza como los estados miembros han incorporado las competencias clave a sus sistemas educativos. Concretamente en España, los cambios normativos ligados al desarrollo de la Ley Orgánica 8/2013, de 9 de diciembre, para la 
mejora de la calidad educativa (LOMCE) han reflejado que la adopción del enfoque por competencias es uno de los motivos que justifican la reforma (Ramírez, 2015).

No obstante, el estudio del fenómeno competencial supone un debate conceptual con profundas implicaciones en el proceso de enseñanza y aprendizaje. Algunas de estas implicaciones hacen del modelo un complejo sistema, que atañe a elementos como la organización escolar, la formación docente, el desarrollo curricular o el propio desempeño docente en la planificación, la metodología o la evaluación (Monarca y Rappoport, 2013, Ramírez, 2015).

Ahora bien, este complejo constructo debe contemplar como piedra angular, esencial y activa del proceso de enseñanza y aprendizaje al alumno. Por tanto, parece necesario impulsar la investigación, conceptualización y estructuración de los cambios unidos al enfoque competencial, con el fin de posibilitar el desarrollo de competencias del alumno, en favor de su inclusión social, ciudadanía activa y empleo (European Council, 2006).

Desde la aparición de estudios pioneros como el Proyecto Tuning (González y Wagenaar, 2003), las investigaciones en la Educación Superior han proliferado impulsando la adopción del modelo como parte de la cultura educativa universitaria. Sin embargo, el ritmo al que los estudios se suceden en Primaria o Secundaria es mucho menor (por citar algunos, Barrachina y Blasco, 2012; Méndez-Giménez, Sierra y Mañana, 2013; Monarca y Rappoport, 2013; Ramírez, 2015).

En lo que respecta a la enseñanza por competencias, a pesar del alcance e importancia internacional del modelo, algunos de estos estudios han evidenciado un distanciamiento entre las pretensiones del currículo prescrito y lo acontecido en el currículo en acción. Además, sugieren dificultades para avanzar en el proceso de integración del modelo en el desempeño docente imperante en niveles educativos preuniversitarios (Barrachina y Blasco, 2013; Ramírez, 2015).

Por tanto, a pesar de que algunas de estas investigaciones pueden ser referentes para desarrollar futuras líneas de investigación, a través de esta propuesta se pretende proporcionar un referente para el desarrollo de futuros proyectos de investigación que indaguen sobre el modo en que los elementos de la reforma competencial se imbrican en los sistemas educativos, los diferentes niveles de enseñanza y las distintas asignaturas. Además, se busca que este diseño no sólo sirva para indagar sobre el problema de estudio, sino que, al mismo tiempo, reforme la práctica docente, favoreciendo el desarrollo profesional, la formación del profesorado y la inclusión del modelo competencial en la realidad de las aulas. En consecuencia, este estudio tiene como objetivo metaevaluar una investigación evaluativa, indagando sobre el modo en que este estudio influyó en los participantes y pudo contribuir a la integración del modelo competencial en su desempeño docente.

\section{Metodología}

Este estudio siguió el método evaluativo enfocado desde la complementariedad paradigmática (Cook y Reichardt, 1986). Si bien fue desarrollado mayoritariamente con una metodología cualitativa, ésta fue complementada con técnicas cuantitativas. Siguiendo a Castejón (1996), Lukas y Santiago (2004) y Medina y Villar (1995), esta investigación evaluativa fue externa, nomotética criterial, orientada a la toma de decisiones y profesional. Asimismo, se desarrolló en una sucesión de cuatro fases relacionadas entre sí: preliminar, de desarrollo, de continuidad y de valoración. 


\section{Participantes}

Para la selección de la muestra se siguió un muestreo intencional de variación máxima para obtener una muestra heterogénea respetando cierta homogeneidad (Goetz y LeCompte, 1988). Esta muestra fue constituida por seis docentes de Educación Física (cuatro hombres y dos mujeres), de Educación Secundaria Obligatoria (ESO) de la Comunidad de Madrid, cuyos centros educativos estaban situados en municipios de diferente tamaño poblacional y, entre ellos, existía una dispersión espacial entre las diferentes áreas geográficas de la Comunidad de Madrid.

Las características de los participantes en cuanto al sexo, la titularidad del centro, la vinculación con el centro educativo, los años de experiencia docente y el nivel educativo donde ejercen la docencia en Educación Física, se especifican en la Tabla 1.

Tabla 1.

Características de la muestra de la investigación

\begin{tabular}{|c|c|c|c|c|c|}
\hline Participantes & Sexo & $\begin{array}{c}\text { Titularidad del } \\
\text { centro }\end{array}$ & $\begin{array}{c}\text { Vinculación } \\
\text { centro }\end{array}$ & $\begin{array}{c}\text { Años de } \\
\text { experiencia }\end{array}$ & $\begin{array}{c}\text { Nivel } \\
\text { educativo } \\
\text { ESO }\end{array}$ \\
\hline Docente 1 & Hombre & Público & $\begin{array}{c}\text { Destino } \\
\text { definitivo }\end{array}$ & 17 & $3^{\circ} / 4^{\circ}$ \\
\hline Docente 2 & Mujer & Público & $\begin{array}{c}\text { Expectativa de } \\
\text { destino }\end{array}$ & 15 & $1^{\circ} / 3^{\circ}$ \\
\hline Docente 3 & Hombre & Público & $\begin{array}{c}\text { Destino } \\
\text { definitivo }\end{array}$ & 22 & $4^{\circ} / 4^{\circ}$ \\
\hline Docente 4 & Mujer & Público & $\begin{array}{c}\text { Destino } \\
\text { definitivo }\end{array}$ & 21 & $3^{\circ}$ \\
\hline Docente 5 & Hombre & Público & Interino & 1,6 & $2^{\circ} / 3^{\circ} / 4^{\circ}$ \\
\hline Docente 6 & Hombre & Privado & $\begin{array}{c}\text { Contrato } \\
\text { temporal }\end{array}$ \\
\hline
\end{tabular}

\section{Fases de la investigación evaluativa}

Secuenciar los elementos de una investigación en una unidad temporal de acuerdo a objetivos prestablecidos da coherencia al proceso metodológico y clarifica las tareas del evaluador. Por tanto, a continuación se expone la secuenciación de las técnicas e instrumentos en las cuatro fases introducidas anteriormente: preliminar, de desarrollo, de continuidad y de valoración.

Estas fases configuran un sistema interactivo que busca la mejora continua de sus componentes. De hecho, el final de una fase condujo y guío el comienzo de la siguiente, a través de la definición previa de criterios estables que orientan el proceso de investigación, los resultados y la consecuente toma de decisiones. A continuación se desarrollarán estas fases de forma pormenorizada.

En primer lugar, se realizó una fase preliminar (Tabla 2) cuyo objetivo era analizar el uso de las competencias en documentos externos (en el contexto nacional y madrileño) e internos 
(Proyectos de los centros educativos participantes y las propias programaciones de Departamento) mediante una revisión documental y la técnica de análisis de contenido.

Usando términos del modelo CIPP de Stufflebeam y Shinkfield (1987), esta fase permitiría controlar "context" e "inputs" y determinar los criterios de evaluación acerca de la enseñanza por competencias. Este hecho orientó la evaluación en las siguientes fases a partir de la normativa. De este modo, se revisó la normativa que regulaba la contribución a las competencias en Educación Secundaria en la Comunidad de Madrid en el momento en que se desarrolló el estudio (entre otros documentos, Decreto 23/2007, de 10 de mayo, del Consejo de Gobierno, por el que se establece para la Comunidad de Madrid el currículo de la Educación Secundaria Obligatoria).

De este modo, se definieron con precisión los criterios que podrán ser traducidos a conductas observables o cuantificables en las siguientes fases (García-Llamas, 2003). Algunos ejemplos de estos criterios que guiaron el diseño, elaboración y aplicación de instrumentos, así como el análisis de la información, fueron:

- Competencia en Autonomía e Iniciativa Personal: "Promover la participación del alumnado en la organización de proyectos o jornadas relacionados con AFD adecuados a sus necesidades".

- Competencia Social y Ciudadana: "Favorecer la cooperación y el trabajo en equipo".

Los criterios expuestos sirven para ejemplificar el proceso de investigación seguido con el objetivo de favorecer su replicabilidad. No obstante, la actual normativa derivada de la LOMCE exigiría una revisión de sus desarrollos curriculares, para generar nuevos criterios con los que planificar y desarrollar nuevos proyectos de investigación.

La fase preliminar constituye un momento fundamental. Esta fase debe asegurar unos criterios de evaluación de calidad, sea cual sea el objeto o problema de estudio al que se atienda. Además, pretende conocer los propios programas, a través de un análisis de contenido, y los contextos en que serán desarrollados.

Tabla 2.

Resumen de la fase I: preliminar. Evaluación de contexto. Enero-Febrero de 2011

\begin{tabular}{|c|c|c|c|}
\hline FASES & DESCRIPCIÓN & $\begin{array}{l}\text { INSTRUMENTO DE } \\
\text { RECOGIDA DE LA } \\
\text { INFORMACIÓN }\end{array}$ & $\begin{array}{l}\text { TRATAMIENTO DE } \\
\text { LA INFORMACIÓN }\end{array}$ \\
\hline $\begin{array}{c}\text { FASE I. FASE } \\
\text { PRELIMINAR. } \\
\text { Evaluación de } \\
\text { contexto. } \\
\text { Objeto: } \\
\text { - Contextualización }\end{array}$ & $\begin{array}{l}\text { - Conocimiento de la } \\
\text { situación de la enseñanza } \\
\text { por competencias y de las } \\
\text { competencias como } \\
\text { elemento curricular en la } \\
\text { Educación Primaria, } \\
\text { Secundaria y Educación } \\
\text { Superior }\end{array}$ & $\begin{array}{c}\text { Revisión de la } \\
\text { normativa y legislación } \\
\text { vigente en el contexto } \\
\text { nacional en la Educación } \\
\text { Primaria, Secundaria y la } \\
\text { Educación Superior }\end{array}$ & \multirow[t]{2}{*}{ Cualitativo } \\
\hline $\begin{array}{c}\text { de las competencias } \\
\text { en la Educación } \\
\text { Secundaria } \\
\text { Obligatoria. } \\
\text { - Contextualización } \\
\text { de las } \\
\text { programaciones }\end{array}$ & $\begin{array}{l}\text { - Análisis de las demandas } \\
\text { legislativas y normativas } \\
\text { para la contribución y } \\
\text { evaluación de las } \\
\text { competencias en la ESO } \\
\text { en la Comunidad } \\
\text { Autónoma de Madrid. }\end{array}$ & $\begin{array}{c}\text { Revisión de la } \\
\text { normativa y legislación } \\
\text { vigente en ESO en la } \\
\text { Comunidad Autónoma } \\
\text { de Madrid }\end{array}$ & \\
\hline
\end{tabular}




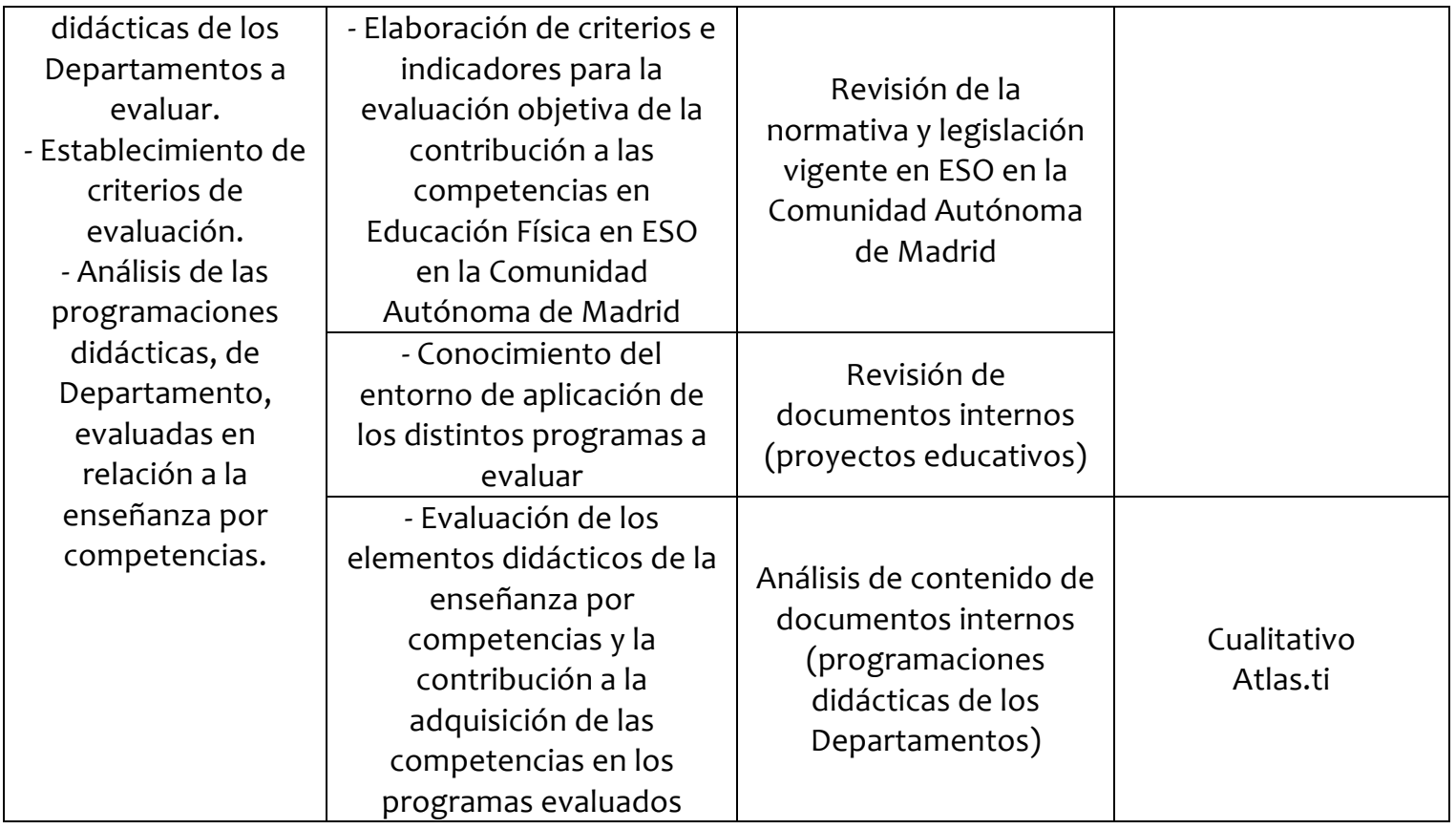

La fase preliminar es la antesala de la segunda fase, de desarrollo. Dicha fase constituye una evaluación de la implementación. Esta evaluación pretende descubrir los errores en los procedimientos empleados por el docente (Colás y Rebollo, 1993).

En esta fase se tradujeron los criterios establecidos en la anterior en criterios observables y cuantificables en el propio contexto de desarrollo de las programaciones, atendiendo a todos los implicados en ellas: docentes y alumnos. Así, el diseño de los instrumentos y la aplicación de técnicas estuvieron fundamentados en aquellos criterios y buscaban resolver las cuestiones de evaluación.

Siguiendo esta serie de premisas metodológicas, durante esta fase se desarrolla una observación naturalista y sistematizada para conocer la contribución a las competencias en la aplicación de las programaciones de los participantes. Esta observación fue aplicada en las sesiones de los profesores evaluados por el evaluador externo y se sistematizó aplicando tres instrumentos: notas de campo, perfiles y escalas de clasificación descriptivas. La observación se complementó con técnicas de grupo de discusión (con los docentes participantes) y encuesta mediante de cuestionario (aplicada a alumnos de los participantes). De este modo, se pretende analizar los criterios de evaluación desde todas las perspectivas posibles: evaluador, docentes y alumnos. La secuenciación de estas técnicas se recoge en la Tabla 3. 
Tabla 3.

Resumen de la fase Il: de desarrollo. Evaluación de la implementación. Marzo-Junio de 2011

\begin{tabular}{|c|c|c|c|}
\hline FASES & DESCRIPCIÓN & $\begin{array}{l}\text { INSTRUMENTO DE } \\
\text { RECOGIDA DE LA } \\
\text { INFORMACIÓN }\end{array}$ & $\begin{array}{l}\text { TRATAMIENTO DE } \\
\text { LA INFORMACIÓN }\end{array}$ \\
\hline \multirow[b]{2}{*}{$\begin{array}{c}\text { Evaluación de la } \\
\text { implementación o de } \\
\text { proceso. }\end{array}$} & $\begin{array}{c}\text { - Elaboración de } \\
\text { instrumentos de } \\
\text { observación externa }\end{array}$ & \multirow{3}{*}{$\begin{array}{c}\text { Observación } \\
\text { naturalista } \\
\text { sistematizada y } \\
\text { estandarizada } \\
\text { mediante notas de } \\
\text { campo, perfil y } \\
\text { escala de } \\
\text { clasificación } \\
\text { descriptiva }\end{array}$} & \multirow{3}{*}{$\begin{array}{c}\text { Cualitativo } \\
\text { (Atlas.ti/Excel) }\end{array}$} \\
\hline & $\begin{array}{l}\text { - Desarrollo de la prueba } \\
\text { piloto de observación } \\
\text { externa y rediseño de } \\
\text { instrumentos de } \\
\text { observación }\end{array}$ & & \\
\hline \multirow{3}{*}{$\begin{array}{l}\text { Objeto: } \\
\text { - Análisis de la enseñanza } \\
\text { por competencias en la } \\
\text { implementación de los } \\
\text { programas de los } \\
\text { docentes participantes } \\
\text { (contribución a las } \\
\text { competencias, } \\
\text { metodología y evaluación } \\
\text { por competencias). }\end{array}$} & $\begin{array}{c}\text { - Ejecución de observación } \\
\text { externa y registro de } \\
\text { sesiones }\end{array}$ & & \\
\hline & $\begin{array}{c}\text { - Elaboración de } \\
\text { cuestionario para la } \\
\text { aplicación de encuesta a } \\
\text { alumnos }\end{array}$ & $\begin{array}{c}\text { Encuesta mediante } \\
\text { cuestionario }\end{array}$ & Cuantitativo (SPSS) \\
\hline & $\begin{array}{c}\text { - Realización de grupo de } \\
\text { discusión con docentes } \\
\text { participantes }\end{array}$ & $\begin{array}{l}\text { Grupo de discusión } \\
\text { grabado en audio }\end{array}$ & Cualitativo (Atlas.ti) \\
\hline $\begin{array}{l}\text { - Conocimientos, } \\
\text { concepciones, } \\
\text { expectativas y formación } \\
\text { del profesorado } \\
\text { participante sobre la } \\
\text { enseñanza por } \\
\text { competencias. }\end{array}$ & $\begin{array}{c}\text { - Aplicación de encuesta } \\
\text { mediante cuestionario } \\
\text { aplicada a alumnos de los } \\
\text { participantes }\end{array}$ & $\begin{array}{c}\text { Encuesta mediante } \\
\text { cuestionario }\end{array}$ & Cuantitativo (SPSS) \\
\hline
\end{tabular}

En una tercera fase, de continuidad (Tabla 4), se aplica una entrevista semiestructurada con los docentes para complementar la información obtenida durante la evaluación de la implementación. De hecho, las entrevistas ofrecen oportunidades para probar y contrastar información (Colás y Rebollo, 1993). Para Lukas y Santiago (2004), las entrevistas sirven de complemento para la observación, en especial, en las investigaciones evaluativas de carácter cualitativo.

Además, durante esta fase, se proporcionaron recomendaciones (toma de decisiones), en función de los resultados obtenidos en las fases anteriores, a través de un informe de evaluación individual, que fue entregado a cada docente por medio de correo electrónico. El informe es un componente fundamental de la investigación evaluativa (Pérez-Juste, 2000).

Por tanto, se desarrolló el mismo atendiendo a las recomendaciones de expertos (Colás y Rebollo, 1993; De Miguel, 2000; Joint Comittee on Standards for Educational Evaluation [JCSEE], 2001; Medina y Villar, 1995) para proporcionar a los participantes los resultados en torno al problema de estudio. Basados en esos resultados, el informe incorporó una serie de recomendaciones para la mejora del desempeño. Con ello, se pretende llegar al currículo en acción y proporcionar la información necesaria para generar cambio. 
Tabla 4.

Resumen de la fase III: de continuidad. Complementación y profundización en la evaluación de la implementación. Junio de 2011-Marzo de 2013

\begin{tabular}{|c|c|c|c|}
\hline FASES & DESCRIPCIÓN & $\begin{array}{l}\text { INSTRUMENTO DE } \\
\text { RECOGIDA DE LA } \\
\text { INFORMACIÓN }\end{array}$ & $\begin{array}{l}\text { TRATAMIENTO DE } \\
\text { LA INFORMACIÓN }\end{array}$ \\
\hline $\begin{array}{l}\text { FASE III. FASE DE } \\
\text { CONTINUIDAD. }\end{array}$ & $\begin{array}{l}\text { - Realización de entrevista } \\
\text { semiestructurada con los } \\
\text { profesores participantes }\end{array}$ & $\begin{array}{l}\text { Entrevista } \\
\text { semiestructurada } \\
\text { grabada en audio }\end{array}$ & Cualitativo (Atlas.ti) \\
\hline $\begin{array}{l}\text { Complementación } \\
\text { de la evaluación de } \\
\text { la implementación. }\end{array}$ & $\begin{array}{c}\text { - Análisis y de los datos de la } \\
\text { fase de desarrollo y de } \\
\text { continuidad }\end{array}$ & \multirow{5}{*}{$\begin{array}{l}\text { Informe de } \\
\text { evaluación }\end{array}$} & $\begin{array}{c}\text { Cuantitativo (SPSS } \\
\text { versión 19) } \\
\text { Cualitativo (Atlas.ti } \\
\text { versión) }\end{array}$ \\
\hline \multirow{4}{*}{$\begin{array}{c}\text { Objeto: } \\
\text { - Profundización } \\
\text { sobre la } \\
\text { información } \\
\text { obtenida de la fase } \\
\text { de desarrollo. } \\
\text { - Creación y } \\
\text { entrega de los } \\
\text { informes de } \\
\text { evaluación } \\
\text { individuales para } \\
\text { los participantes. }\end{array}$} & $\begin{array}{l}\text { - Toma de decisiones para la } \\
\text { construcción del informe }\end{array}$ & & \multirow{4}{*}{$\begin{array}{c}\text { Cuantitativo (SPSS } \\
\text { versión 19) } \\
\text { Cualitativo (Atlas.ti } \\
\text { versión) }\end{array}$} \\
\hline & - Elaboración de los informes & & \\
\hline & $\begin{array}{c}\text { - Revisión de los informes por } \\
\text { expertos: directores del } \\
\text { proyecto }\end{array}$ & & \\
\hline & $\begin{array}{c}\text { - Corrección de los informes y } \\
\text { envío de los mismos a los } \\
\text { participantes mediante correo } \\
\text { electrónico }\end{array}$ & & \\
\hline
\end{tabular}

En ese sentido, la fase de continuidad vuelve a ser el punto de partida para la fase final de esta investigación evaluativa. Dicha fase se encuentra precedida de la elaboración del informe de evaluación, que recogen la toma de decisiones en función de los criterios que han dado coherencia a todo el proceso.

Así, una vez entregados y leídos los informes por los docentes participantes, en la fase de valoración (Tabla 5) se recogieron narrativas de los evaluados. Esta fase se identifica con una metaevaluación o evaluación de la investigación evaluativa (Tejedor, 2000).

Al respecto, se pretende evaluar el proceso, conociendo la incidencia que pudo tener en la formación, las concepciones, las expectativas y el desempeño de los evaluados. Asimismo, el hecho de que los profesores participantes valoraran el método es una manera de incrementar el rigor, asegurando el estándar de utilidad, sobre el seguimiento e impacto de la evaluación (JCSEE, 2001).

El uso de las narrativas ha sido defendido como método para integrarlo con otras técnicas de investigación para afrontar la naturaleza compleja, multidimensional y cambiante de la realidad educativa (Sparkes, 2003).

A continuación se detalla el proceso de recogida y análisis de estas narrativas, pues ha permitido valorar este proceso de investigación y obtener los resultados que se exponen en este artículo. Se recogieron cuatro cuestionarios abiertos que pretendían generar narrativas de los seis docentes participantes en este estudio. Los cuestionarios fueron guiados por medio de preguntas de evocación abiertas de modo que incitaran a la expresión de las ideas 
y concepciones de los participantes. Los datos fueron analizados de forma inductiva utilizando Atlas.ti, en su versión 5.0., siguiendo la "Teoría Fundamentada" de Glaser y Strauss (1967).

Tabla 5.

Resumen de la fase IV: de valoración. Metaevaluación. Marzo-Mayo de 2013

\begin{tabular}{|c|c|c|c|}
\hline FASES & DESCRIPCIÓN & $\begin{array}{l}\text { INSTRUMENTO DE } \\
\text { RECOGIDA DE LA } \\
\text { INFORMACIÓN }\end{array}$ & $\begin{array}{l}\text { TRATAMIENTO DE } \\
\text { LA INFORMACIÓN }\end{array}$ \\
\hline $\begin{array}{c}\text { FASE IV. FASE DE } \\
\text { VALORACIÓN. } \\
\text { Metaevaluación. } \\
\text { Objeto: } \\
\text { - Valoración de la aceptación y } \\
\text { utilidad de la toma de } \\
\text { decisiones } \\
\text { - Conocimiento de los cambios } \\
\text { didáctico-pedagógicos en la } \\
\text { intervención docente de los } \\
\text { participantes en relación a la } \\
\text { enseñanza por competencias. } \\
\text { - Conocimiento de los cambios } \\
\text { en la formación y en las } \\
\text { expectativas de los } \\
\text { participantes con respecto a la } \\
\text { enseñanza por competencias. }\end{array}$ & $\begin{array}{c}\text { - Elaboración de } \\
\text { cuestionarios } \\
\text { abiertos, revisión por } \\
\text { parte de los } \\
\text { investigadores del } \\
\text { estudio y } \\
\text { reelaboración } \\
\text { definitiva de los } \\
\text { mismos } \\
\\
\text { - Aplicación de los } \\
\text { cuestionarios a los } \\
\text { participantes }\end{array}$ & $\begin{array}{l}\text { Narrativas } \\
\text { mediante } \\
\text { cuestionarios } \\
\text { abiertos }\end{array}$ & $\begin{array}{l}\text { Cualitativo } \\
\text { (Atlas.ti) }\end{array}$ \\
\hline
\end{tabular}

En definitiva, este proceso de evaluación abogó por un diseño que mantenga unos criterios estables, previamente definidos, en base a referentes normativos que regulan el objeto de evaluación en el contexto de investigación. Estos criterios configuran la base para la construcción y aplicación de instrumentos, cuya información aportada es tratada de forma cuantitativa o cualitativa. Estos resultado son, en consecuencia, la base para orientar la toma de decisiones (informe de evaluación individual). Una toma de decisiones y un proceso que, por otro lado, es metaevaluado y atiende a los implicados en la implementación del programa (profesores participantes y alumnos), valorando su impacto y pertinencia (Figura 1). 


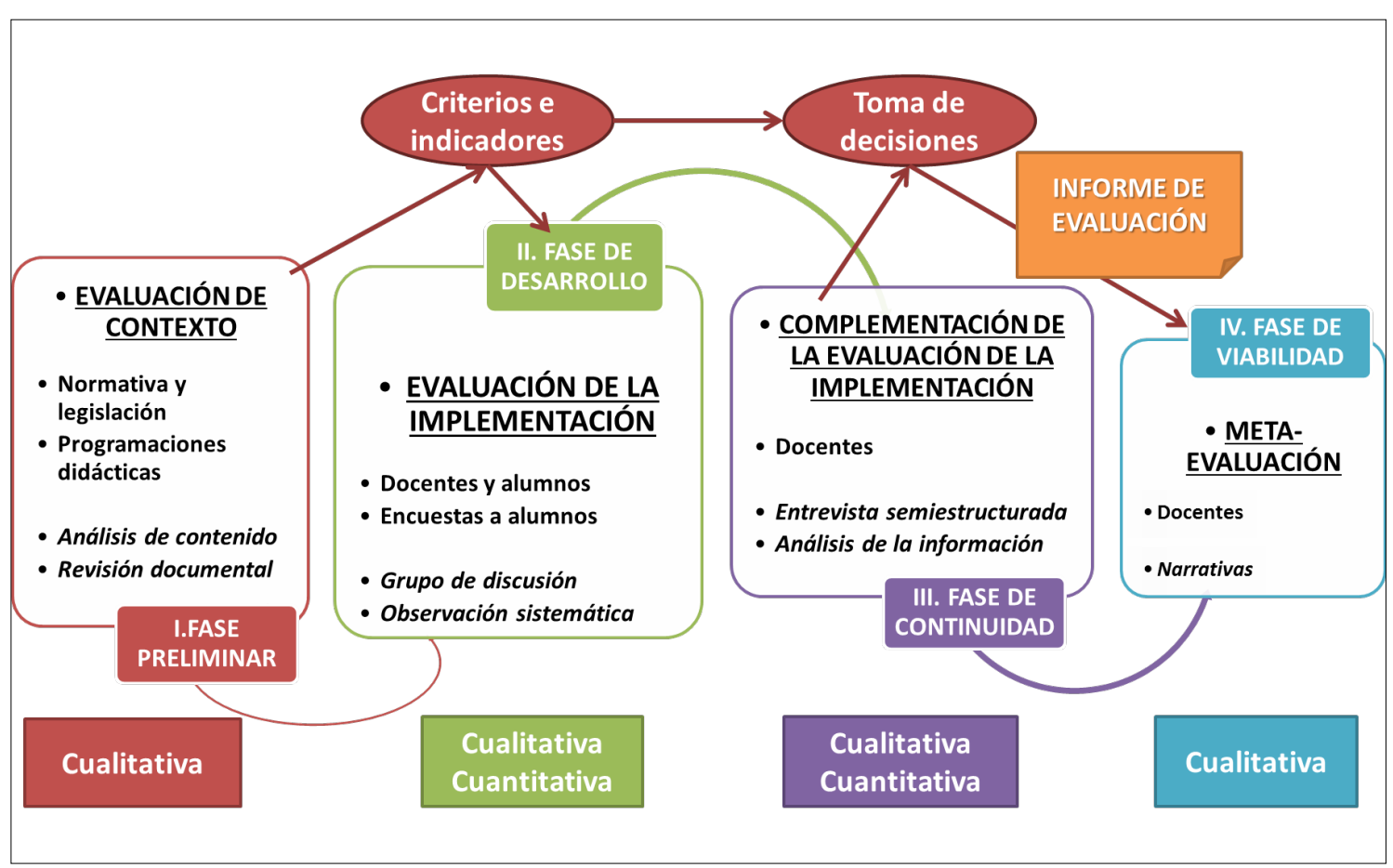

Figura 1. Resumen del proceso de investigación evaluativa

\section{Criterios de credibilidad y rigor}

Esta investigación atendió a criterios de aplicabilidad, confirmabilidad, dependencia y credibilidad (Bisquerra, 1989; Colás y Buendía, 1992; Pérez-Serrano, 1994). Para la consecución de estos criterios: se recopilaron datos procedentes de abundantes técnicas (entrevistas, grupos de discusión, cuestionarios abiertos, observación, análisis de contenido y encuestas); se realiza una descripción detallada del proceso; se utilizaron descriptores de baja inferencia (registros en audio de entrevistas y grupo de discusión o definición concreta de los indicadores de observación basados en la normativa); se desarrolló una prueba piloto de observación para garantizar la viabilidad de la misma y reducir la reactividad de los observados; y se desarrolló una triangulación de lugares (los participantes pertenecían a diferentes DAT de la Comunidad de Madrid), metodológica (a través del empleo de dos o más técnicas) y de fuentes (atendiendo a todos los implicados en el programa: documentos, docentes, alumnos y el propio investigador) y de investigador (a través de la discusión, supervisión y participación de varios investigadores en el estudio).

\section{Resultados. Metaevaluación de los docentes participantes}

Para Pérez-Juste (2006) el mero hecho de evaluar genera cambios, positivos o negativos. Por tanto, una vez presentado el método con suficiente detenimiento, se indaga a continuación sobre la pertinencia e influencia del mismo desde la perspectiva de los participantes (objetivo de este artículo).

A lo largo del artículo se han resaltado dos aspectos relevantes: la intención de la evaluación de generar cambio y dotar a la misma de una orientación práctica y la pretensión de contribuir a que el currículo prescrito en base a la enseñanza por competencias llegue a la práctica. Para valorar la consecución de estos propósitos se recoge a continuación la metaevaluación de este diseño metodológico. Esta valoración fue realizada por los 
participantes a través de sus narrativas, que fueron generadas mediante cuestionarios abiertos y utilizadas en la última fase de la investigación. El análisis de las narraciones condujo a la obtención de los siguientes resultados.

Los docentes valoraron positivamente la investigación evaluativa. Así lo sugieren las siguientes citas:

- "Me parece muy interesante la valoración realizada porque me permite tener una visión y valoración externa de mi trabajo. Este análisis exterior me aporta un feedback muy enriquecedor como profesional y me ayuda a reflexionar sobre el desarrollo de mi labor docente." (Docente 2).

- "Me parece interesante, es necesario un análisis y evaluación de la práctica docente que actualmente no se hacen." (Docente 4).

- "En lo referente a mi consideración de mejoría que me ha podido proporcionar la investigación, tengo que afirmar con rotundidad que ha sido un descubrimiento muy positivo para mis clases y para la percepción que muchos alumnos tenían de la asignatura..." (Docente 6).

En cuanto a la influencia que el proceso tuvo en los participantes parece que incrementó el interés por el modelo competencial y la reflexión sobre su práctica educativa:

- “Considero que, fundamentalmente, esta investigación ha contribuido a incrementar considerablemente mi interés por conocer más sobre la forma de trabajo por competencias y, tras leer el informe sobre mi docencia, a replantear y mejorar mi actividad docente con la finalidad de hacerla más beneficiosa para mi alumnado." (Docente 2).

Ahora bien, las opiniones de los docentes fluctúan entre aquellas que afirman que su forma de trabajo cambió sustancialmente y el trabajo competencial se convirtió en un referente en su práctica educativa (como el Docente 6) y aquellos que reconocen una influencia ligera o baja en su labor (como el Docente 2 y 3 ):

- "Respecto al tratamiento de las competencias básicas, he de decir que después de participar en el proyecto han sido protagonistas en casi todas mis clases." (Docente 6).

- “... mi metodología ha variado ligeramente e intenta centrarse más en el trabajo participativo del alumno y se ha incrementado considerablemente el trabajo interdisciplinar,..." (Docente 2).

- "La ayuda recibida se concreta en afianzar la línea educativa que llevo a cabo, aunque, seguramente tendría que reflejarlo de manera más evidente en la programación" (Docente 3).

Esta influencia se manifestó mayoritariamente en cambios en el uso de metodologías más participativas y en cambios en las nuevas programaciones diseñadas. Así se recoge en las siguientes citas:

- "Mediante una nueva programación que incluían muchos contenidos y unidades didácticas iguales a la anterior, pero, con la diferencia, de que en un gran número de sesiones de la misma ha existido una metodología más cercana a lo requerido por las competencias, con un alumnado más participativo, con una dinámica donde el profesor era una figura guía y de asesoramiento y con unos objetivos encaminados más a formación integral y completa del alumnado 
que a los que las propias prácticas (bien sean juegos, deportes, ejercicios, etc.)." (Docente 6).

- “Creo que sí se han utilizado metodologías más activas (...) independientemente de haber programado de forma explícita el tratamiento de las competencias básicas." (Docente 3 ).

- "Por ello, mis actividades son mucho más prácticas en las que mucha responsabilidad recae sobre en el alumnado en la preparación de clases, en la gestión y el funcionamiento." (Docente 6).

- "Creo que sí se han utilizado metodologías más activas y se ha fomentado la participación del alumnado..." (Docente 4).

Además, el proceso pareció favorecer la formación de alguno de los participantes, incrementando los conocimientos sobre el modelo competencial:

- "Con este trabajo de investigación, he incrementado ligeramente mi conocimiento sobre el trabajo por competencias..." (Docente 2).

No obstante, la influencia en la formación no fue notable para todos los profesores. Algunos docentes expresaron la necesidad de seguir formándose y encontrar apoyos para afianzar el uso del modelo en sus clases. Algunas consideraciones manifiestan que el proceso pudo seguir facilitando su progreso y mejorando su formación:

- "Debo mencionar que, en el apartado de evaluación, sigo teniendo serias dificultades; y es que, no he encontrado aún la forma de poder asignar valores numéricos a la consecución de ciertas competencias." (Docente 6).

- "Las dudas respecto a los cambios que supone trabajar por competencias no se han despejado..." (Docente 3 ).

- “... sigo considerando muy necesaria una mayor formación al respecto mediante seminarios, talleres o grupos de trabajo con otros profesionales." (Docente 2).

Ahora bien, la detección de estas deficiencias formativas, llevó a algunos participantes a impulsar su formación permanente en principios del modelo competencial, como el uso de metodologías activas. Los docentes 3 y 4 afirmaron haber participado en un curso de aprendizaje cooperativo:

- " "Sí hemos hecho un curso de aprendizaje cooperativo que puede modificar el 'modus operandis' de la función docente; aunque sea poco a poco" (Docente 3).

- "Durante el curso he realizado un curso de aprendizaje cooperativo" (Docente 4).

Además, es reseñable que la participación en estos cursos, en el caso de la docente 4 se ha concretado en la aplicación de actividades con metodologías activas y, por tanto, prácticas docentes acordes al modelo competencial. A continuación se recoge algunas de estas actividades de la Docente 4:

- “El examen de la $1^{\text {a }}$ evaluación de la ESO de $3^{\circ}$ de lo planteé con una actividad en equipo. Preparación del tema con esquemas, 4 por grupo, expertos preparan parte del tema, explican al resto del grupo y entre todos elaboran un mural."

- "Corrección de técnica de deportes por parejas" 


\section{Discusión y conclusiones}

Si bien los resultados de una investigación cualitativa no pretenden ser generalizables, este proceso de investigación evaluativa en particular fue valorado positivamente por los participantes e influyó en su práctica docente, lo que podría justificar la propuesta de este diseño para explorar el problema, el modelo competencial en Educación Física en Secundaria, así como favorecer la formación del profesorado y el progreso en su desempeño profesional. Al respecto, no se trata de universalizar la propuesta, sino de transmitirla, justificándola a través de su metaevaluación, para que pueda ser útil en su globalidad o en alguno de sus elementos a otros investigadores, instituciones o centros educativos para explorar el problema de este estudio en otros contextos.

De hecho, según Tejedor (2003) la evaluación es una herramienta al servicio de la formación de profesionales en respeto con el servicio que reportan a la sociedad. De hecho, la evaluación de la práctica docente ha surgido con un gran énfasis e interés en el contexto nacional para responder a las exigencias legislativas, mejorar la calidad de las instituciones y el desarrollo profesional del docente (Muñoz, Ríos y Abalde, 2002).

En este sentido, parece que investigación, evaluación y desempeño docente son términos vinculados a la calidad educativa. Desde este punto de vista, investigar supone favorecer la formación del profesorado, si éstos participan o colaboran en procesos de investigación. La investigación puede favorecer el desarrollo profesional de los docentes mejorando la calidad de su desempeño y, por ende, de la educación. De hecho, la cualificación de la profesión docente y calidad educativa son dos variables con una alta correlación y el desempeño docente se considera un factor preponderante en la calidad educativa y es evaluado para mejorar ésta (Montenegro, 2007).

Además, estos resultados sugieren que esta investigación evaluativa tuvo consecuencias en la formación de los participantes, atendiendo a los requerimientos sobre este nuevo desempeño que se le ha encomendado: la enseñanza por competencias (Ramírez, 2015). Desde el punto de vista de la investigación evaluativa, podría afirmarse que la evaluación fue útil y estos resultados dotan de credibilidad a la investigación realizada (Cabrera, 2000; De Miguel, 2000; JCSEE, 2001; Mateo, 2000).

Estos planteamientos son compartidos por Campos-Izquierdo (2010) en el campo de la actividad física y el deporte, quien sostiene que es preciso que se investigue y evalúe el desempeño profesional de los recursos humanos para mejorar la calidad de los servicios que presten a la sociedad. Asimismo, concretamente en el campo de la Educación Física, Campos-Izquierdo, González-Rivera y Jiménez-Beatty (2012) consideran primordial la formación permanente del profesorado para desempeñar adecuadamente. En la misma línea, Imbernón (2002) asevera que la investigación es formación. De hecho, para Fernández (2003), la investigación es un proceso educativo para el propio profesor, que le proporciona destrezas y le enriquece en las dimensiones personales básicas para el ejercicio de su profesión.

Por tanto, observando la rápida consolidación del modelo competencial (Fernández-Balboa, 2008), surge la necesidad de evaluar al docente sobre la aplicación de la enseñanza por competencias. Así, evaluar al docente sobre este objeto, teniendo como objetivo la mejora y el perfeccionamiento de su actividad, puede promover la consolidación de un modelo educativo que, en su diseño, pretende ayudar al alumno en su inclusión social y profesional.

Sin embargo, a pesar del valor aparente del modelo y su creciente afianzamiento con la aparición de la Ley Orgánica 8/2013, de 9 de Diciembre, para la mejora de la calidad educativa, las líneas de investigación y actuación para la adopción del fenómeno de las 
competencias no han proliferado en los niveles preuniversitarios. Al mismo tiempo, los estudios que aparecen advierten que el modelo no llega a la práctica (Barrachina y Blasco, 2012; Monarca y Rappoport, 2013). Además, ni la experiencia docente ni la formación inicial son suficientes para acometer el proceso de operativización de las competencias (Ramírez, 2015).

En consecuencia, el desarrollo de métodos de investigación orientados a la práctica, como el que se presenta en este estudio, pueden ser útiles desde una doble perspectiva: la indagación sobre un problema de estudio de gran relevancia y poco explorado en el contexto no universitario y la influencia en la práctica docente, de modo que facilite la adopción de un modelo que no llega a la práctica.

No obstante, los resultados de esta metaevaluación sugirieron que los participantes no se encontraban plenamente preparados para acometer el modelo tras la investigación, persistiendo alguna deficiencia formativa. Estos resultados advierten, también, de las limitaciones de una evaluación externa, no realizada desde premisas colaborativas ni cíclicas, que permitan progresar en la formación y desempeño del profesorado de forma continua. Por tanto, se plantea la posibilidad de reflexionar y plantear futuros proyectos de investigación con seguimientos más profundos de los participantes, que faciliten la continuidad y el progreso constante en su desempeño.

En este sentido, el método evaluativo adquiere un carácter flexible y dinámico que facilita la replicabilidad y adaptación del proceso en diferentes contextos, al mismo tiempo que se ajusta a una realidad educativa cambiante y compleja. La investigación evaluativa puede atender a diferentes objetos, admite la complementariedad metodológica, el uso de diversos instrumentos y favorece la participación de todos los implicados en el proceso de enseñanza y aprendizaje, pudiendo seguir modelos de investigación-acción con evaluadores internos (Lukas y Santiago, 2004). De hecho, diseños evaluativos como los de Díez (2013) sugieren que los procesos de investigación evaluativa colaborativas pueden servir a la mejora de la calidad de las organizaciones educativas. Por tanto, la indagación en el problema desde planteamientos evaluativos colaborativos puede conformar una posible futura línea de investigación.

\section{Referencias}

Barrachina, J., \& Blasco, J. E. (2012). Análisis del desarrollo de las competencias básicas en el currículum de la Educación Física en la ESO en la Marina Baixa. Un estudio de caso. Apunts: Educación física y deportes, 110, 36-44.

Bisquerra, R. (1989). Métodos de investigación educativa. Barcelona: Ediciones Ceac.

Cabrera, F. (2000). Evaluación de la formación. Madrid: Síntesis.

Campos-Izquierdo, A. (2010). Dirección de recursos humanos en las organizaciones de actividad física y deporte. Madrid: Síntesis.

Campos-Izquierdo, A., González-Rivera, M.D., \& Jiménez-Beatty, E. (2012). El perfil profesional del profesorado de Educación Física en Educación Secundaria en la Comunidad Valenciana. Educación XX1, 15 (1), 135-155

Castejón, F. J. (1996). Evaluación de programas en educación física. Madrid: Gymnos.

Colás, M., \& Buendía, L. (1992). Investigación educativa. Sevilla: Alfar.

Colás, M., \& Rebollo, M.A. (1993). Evaluación de programas. Una guía práctica. Sevilla: KRONOS. 
Cook, T., \& Reichardt, C. (1986) Métodos cualitativos y cuantitativos en investigación evaluativa. Madrid: Morata.

Díez, E.J. (2013). Investigación-acción participativa: el cambio cultural con la implicación de los participantes. Revista Electrónica Interuniversitaria de Formación del Profesorado, 16 (3), 115-131.

De Miguel, M. (2000). La evaluación de programas sociales: fundamentos y enfoques teóricos. Revista de investigación educativa, 18 (2), pp. 289-317.

Decreto 23/2007, de 10 de mayo, del Consejo de Gobierno, por el que se establece para la Comunidad de Madrid el currículo de la Educación Secundaria Obligatoria. Boletín Oficial de la Comunidad de Madrid de 29 de Mayo de 2007

European Commission, EACEA y Eurydice (2012). Developing Key Competences at School in Europe: Challenges and Opportunities for Policy. Eurydice Report. Luxembourg: Publications Office of the European Union.

European Council (2006). Recommendation of the European Parliament and of the Council, of 18 December 2006, on key competences for lifelong learning. Official Journal of the European Union. L394 de 30/12/2006, 10-18.

Fernández, M. (2003). La profesionalización del docente. Perfeccionamiento. Investigación en el aula. Análisis de la práctica ( $4^{\mathrm{a}}$ ed.). Madrid: Siglo XXI de España Editores.

Fernández-Balboa, J.M. (2008). ¿Debemos contribuir a la convergencia europea a través de un modelo de formación de futuros docentes por competencias? Reflexiones desde una perspectiva sociocrítica. Tándem. Didáctica de la Educación Física, 26, 19-33.

García-Llamas, J. L. (2003). Métodos de investigación en educación. Volumen II. Investigación cualitativa y evaluativa. Madrid: UNED.

Glaser, B., \& Strauss, A. (1967). The discovery of Grounded Theory. Strategies for qualitative research. Aldine de Gruyter: New York.

González, J., \& Wagenaar, R. (2003). Tuning educational structures in Europe project (Informe final. Fase Uno). Bilbao: Universidad de Deusto.

Imbernón, F. (2002). La investigación educativa como herramienta de formación del profesorado. Reflexión y experiencias de investigación educativa. Barcelona: GRAO.

JCSEE (2001). Estándares de evaluación de personal. Cómo evaluar sistemas de evaluación de educadores. Bilbao: Mensajero.

Ley Orgánica 8/2013, de 9 de diciembre, para la mejora de la calidad educativa. Boletín Oficial del Estado de 10 de Diciembre de 2013.

Lukas, J. F., \& Santiago, K. (2004). Evaluación educativa. Madrid: Alianza Editorial.

Martínez-Mediano, C. (2004). Técnicas e instrumentos de recogida y análisis de datos. Madrid: UNED.

Mateo, J. (2000). La evaluación del profesorado y la gestión de la calidad de la educación. Hacia un modelo comprensivo de evaluación sistemática de la docencia. Revista de Investigación Educativa, 18 (1), 7-34.

Medina, A., \& Villar, L.M. (1995). Evaluación de programas educativos, centros y profesores. Madrid: Editorial Universitas. 
Méndez-Giménez, A., Sierra, B., \& Mañana, J. (2013). Percepciones y creencias de los docentes de primaria del Principado de Asturias sobre las competencias básicas. Revista de Educación, 362, 737-761.

Monarca, H., \& Rappoport, S. (2013). Investigación sobre los procesos de cambio educativo: El caso de las competencias básicas en España. Revista de Educación, Extraordinario, 54-78.

Montenegro, I.A. (2007). Evaluación del desempeño docente. Fundamentos, modelos e instrumentos ( $2^{\mathrm{a}}$ ed.). Colombia: Magisterio.

Muñoz, J.M., Ríos, M.P., \& Abalde, E. (2002). Evaluación Docente vs. Evaluación de la Calidad. Revista Electrónica de Investigación y Evaluación Educativa, 8 (2), 103-134.

Pérez-Juste, R. (2000). La evaluación de programas educativos: conceptos básicos, planteamientos generales y problemática. Revista de investigación educativa, 18 (2), pp. 261-287.

Pérez-Juste, R. (2006). Evaluación de programas educativos. Madrid: La Muralla.

Pérez-Serrano, G. (1994). Investigación cualitativa. Retos e interrogantes. II. técnicas y análisis de datos. Madrid: La Muralla.

Ramírez, A. (2015). La formación del profesorado de Educación Primaria ante las competencias básicas. Revista Electrónica Interuniversitaria de Formación del Profesorado, 18 (3), 199-214.

Real Decreto 1631/2006, de 29 de diciembre, por el que se establecen las enseñanzas mínimas correspondientes a la Educación Secundaria Obligatoria. Boletín Oficial del Estado de 5 de Enero de 2007.

Sparkes, A. (2003). Investigación narrativa en la Educación Física y el deporte. Ágora para la Educación Física y el Deporte, 2 (3), 51-60.

Stufflebeam, D., \& Shinkfield, A. (1987). Evaluación sistemática. Barcelona: Paidós/MEC.

Tejedor, F.J. (2000). El diseño y los diseños en la evaluación de programas. Revista de Investigación Educativa, 18 (2), 319-339.

Tejedor, F.J. (2003). Un modelo de evaluación del profesorado universitario. Revista de Investigación Educativa, 21 (1), 157-182. 